\title{
Randomization Ratio
}

National Cancer Institute

\section{Source}

National Cancer Institute. Randomization Ratio. NCI Thesaurus. Code C92431.

The quotient of quantities of trial subjects within treatment or control groups using an element of chance to determine the assignments, in order to reduce bias. 